\title{
Ex-situ and In-situ Analysis of MoVTeNb Oxide by Aberration-Corrected Scanning Transmission Electron Microscopy
}

\author{
Pinghong $\mathrm{Xu}^{1}$, Maricruz Sanchez-Sanchez ${ }^{2}$, Andre C. Van Veen ${ }^{2}$, Nigel D. Browning ${ }^{3}$, Johannes A. \\ Lercher $^{2,3}$ \\ ${ }^{1}$ Department of Chemical Engineering and Materials Science, University of California, Davis, Davis, \\ USA. \\ ${ }^{2}$ Department of Chemistry and Catalysis Research Center, Technische Universitat Munchen, 85748 \\ Munich, Germany. \\ ${ }^{3}$ Physical Sciences Laboratory, Pacific Northwest National Laboratory, 902 Battelle Boulevard, \\ Richland, WA 99352, USA.
}

Short chain olefins, especially ethylene and propylene are very important industrial raw materials and are of increasing demand worldwide. The abundance of C2-C3 light alkanes in shale gas makes production of these olefins from oxidative dehydrogenation (ODH) one of the most attractive alternatives to industrial processes [1]. Among all the catalysts for C2-C3 light alkane ODH reactions, the two-phase MoVTeNb oxide system has shown great promise [1]. Much attention has been devoted to the analysis of the crystallography structure of catalytic active M1 phase. However, the nature of the surfaces where the reactions take place and their roles in catalysis have not been resolved. Additionally, it has been reported that surfaces of the M1 phase undergo changes during ODH reactions, which would affect the catalytic activity of the system [2]. Therefore, it is also very important to perform the in situ experimental observations under relatively realistic conditions for a fundamental understanding of the outstanding catalytic performance of this material.

Here, we report an identification of the nature of crystalline termination of the M1 phase at atomic scale using an aberration-corrected scanning transmission electron microscope (STEM). Figure 1 shows a typical M1 phase particle viewed in the crystal growth direction (the [001] orientation). Based on the analysis of over 50 particles, it is shown that the lateral surfaces of these rods are faceted and the most preferential lateral facets have been determined. The configuration of these facets means it is possible to quantify amount of ODH active sites exposed per area on each facet. Moreover, statistical analysis of the proposed active sites exposure in the M1 phase particles with different morphologies agrees very well with experimental data, showing a $\sim 30 \%$ higher activity of the small rounded M1 phase rods compared to the large flattened ones. These results demonstrate that morphology has a large impact on catalytic activity of the MoVTeNb oxide system. Simulations are underway to determine the energies of those surface facets with slightly different configurations, which is essential for fundamental understanding of termination mechanism of material of such complex structures.

Direct imaging of structural changes in the M1 phase was performed under an oxidative atmosphere by in-situ TEM technique using a Protochip heating holder in an environmental TEM. An M1 phase particles was heated to $350{ }^{\circ} \mathrm{C}$ in 10 mbar oxygen/argon $(23 \% / 77 \%)$ showed that tellurium units disappeared from hexagonal channels while the crystal framework remained unaffected. Control experiments with sample heated to the same temperature without oxygen showed no such effects. The crystalline structure was damaged within seconds. These results indicate that oxygen stabilizes the MoVTeNb oxide structure at elevated temperature. Further in-situ experiments using a gas stage holder 
which allows much higher partial pressure (above 1 bar) are underway to investigate the gas pressure effects [3].

\section{References:}

[1] F. Cavani et al., Catalysis Today 127 (2007), 113-131.

[2] M. Hävecker et al., Journal of Catalysis 285 (2012) 48-60

[3] This work is supported by the U.S. Department of Energy Grant No. DE-FG02-03ER46057. The research described in this paper is part of the Chemical Imaging Initiative at PNNL under Contract DEAC05-76RL01830 operated for DOE by Battelle. It was conducted under the Laboratory Directed Research and Development Program at PNNL, a multiprogram national laboratory operated by Battelle for the U.S. Department of Energy. A portion of the research was performed using EMSL, a national scientific user facility sponsored by the Department of Energy's Office of Biological and Environmental Research and located at Pacific Northwest National Laboratory.
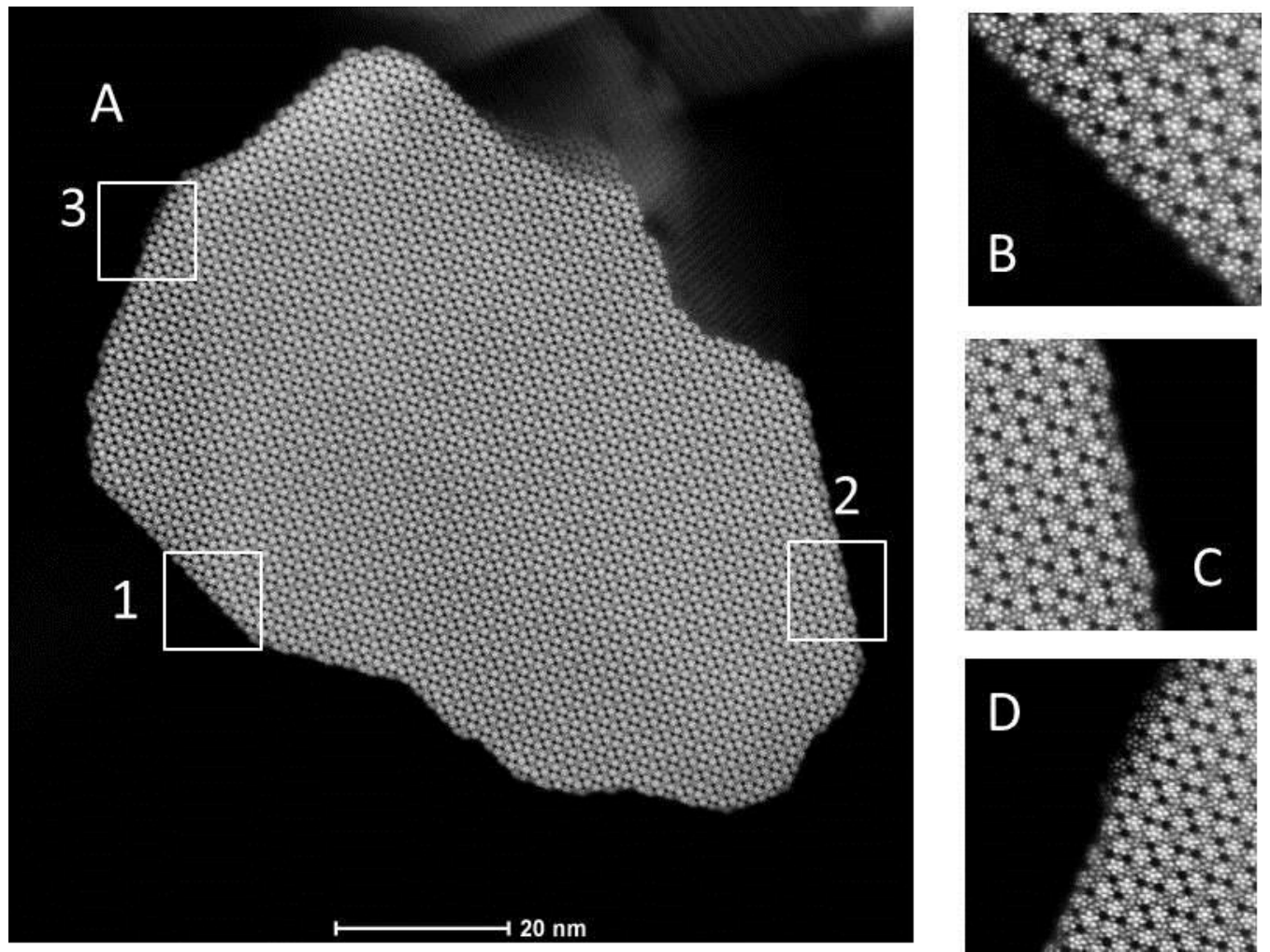

Figure 1. (A) Unprocessed STEM image showing nicely crystalline and faceted surface of M1 phase. (B-D) Magnified view of rectangular areas 1-3 in (A) respectively, showing different facets configurations. 